\title{
Breakfast and exercise contingently affect postprandial metabolism and energy balance in physically active males
}

\author{
Javier T. Gonzalez ${ }^{1 *}$, Rachel C. Veasey ${ }^{1}$, Penny L. S. Rumbold ${ }^{2}$ and Emma J. Stevenson ${ }^{1}$ \\ ${ }^{1}$ Brain, Performance and Nutrition Research Centre, School of Life Sciences, Northumbria University, Northumberland \\ Building, Newcastle upon Tyne NE1 8ST, UK \\ ${ }^{2}$ Department of Sport and Exercise Sciences, School of Life Sciences, Northumbria University, Northumberland Building, \\ Newcastle upon Tyne NE1 8ST, UK \\ (Submitted 30 July 2012 - Final revision received 4 October 2012 - Accepted 14 November 2012 - First published online 23 January 2013)
}

\section{Abstract}

The present study examined the impact of breakfast and exercise on postprandial metabolism, appetite and macronutrient balance. A sample of twelve (blood variables $n$ 11) physically active males completed four trials in a randomised, crossover design comprising a continued overnight fast followed by: (1) rest without breakfast (FR); (2) exercise without breakfast (FE); (3) breakfast consumption (1859 kJ) followed by rest (BR); (4) breakfast consumption followed by exercise (BE). Exercise was continuous, moderate-intensity running (expending approximately $2.9 \mathrm{MJ}$ of energy). The equivalent time was spent sitting during resting trials. A test drink (1500 kJ) was ingested on all trials followed 90 min later by an ad libitum lunch. The difference between the BR and FR trials in blood glucose time-averaged AUC following test drink consumption approached significance (BR: 4.33 (SEM 0.14) $v$. FR: 4.75 (SEM 0.16) mmol/l; $P=0 \cdot 08$ ); but it was not different between FR and FE (FE: 4.77 (SEM 0.14) mmol/l; $P=0.65)$; and was greater in BE $(\mathrm{BE}$ : $4.97(\mathrm{SEM} 0 \cdot 13) \mathrm{mmol} / \mathrm{l}) v$. BR $(P=0.012)$. Appetite following the test drink was reduced in $\mathrm{BR} v$. FR $(P=0.006)$ and in $\mathrm{BE} v$. FE $(P=0 \cdot 029)$. Following lunch, the most positive energy balance was observed in BR and least positive in FE. Regardless of breakfast, acute exercise produced a less positive energy balance following ad libitum lunch consumption. Energy and fat balance is further reduced with breakfast omission. Breakfast improved the overall appetite responses to foods consumed later in the day, but abrogated the appetite-suppressive effect of exercise.

Key words: Appetite: Fasted state: Glycaemia: Fat oxidation

Regular breakfast consumption has been inversely associated with $\mathrm{BMI}^{(1)}$, yet it is not clear whether this association is due to differences in energy expenditure, metabolism or energy intake. Although the ostensible benefits of regular breakfast consumption could be due to improved diet composition with breakfast cereals $^{(1)}$, rather than meal pattern per se, acute consumption of breakfast can enhance glucose tolerance, insulin sensitivity and subjective and physiological satiety responses to a test $\operatorname{drink}^{(2)}$.

A recent position statement concluded that further research is required in regular exercisers with regards to meal pattern, metabolism and appetite regulation ${ }^{(3)}$, as research in exercising individuals in this area is sparse. However, this population do use diet/exercise strategies, such as training in the fasted state, to control body fat/mass and improve metabolic adaptations to training ${ }^{(4)}$. Exercise attenuates adverse dietary outcomes such as fat-induced glucose intolerance ${ }^{(5)}$, and the nutritional state in which exercise is performed can modulate the magnitude of these improvements ${ }^{(5)}$. Exercise in the fasted state results in a greater reliance on fat as a substrate ${ }^{(6)}$ and has led to its use as a tool to reduce body fat by athletes ${ }^{(4)}$. Training in the fasted state also leads to enhanced fat transporter protein mRNA content ${ }^{(5)}$, mitochondrial enzyme activity and maximal aerobic capacity ${ }^{(7)}$, making exercise in the fasted state an attractive proposition for both recreational and elite athletes. On the other hand, high carbohydrate availability during exercise training may result in improved body composition, as gains in fat-free mass are amplified, whilst fat loss is similar ${ }^{(8)}$. Hence, although there is a suggestion that exercise in the fasted state can maximise some benefits already associated with exercise, ensuing effects on appetite and metabolism are not entirely clear.

The regulation of acute energy balance involves (not exclusively) the exposure and sensitivity to the circulating

\footnotetext{
Abbreviations: $\mathrm{AUC}_{\mathrm{INS} / \mathrm{GLU}}$, serum insulin AUC to blood glucose AUC ratio; BE, overnight fast followed by breakfast and exercise; BR, overnight fast followed by breakfast and rest; FE, overnight fast followed by exercise without breakfast; FR, overnight fast followed by rest without breakfast; GLP-1,

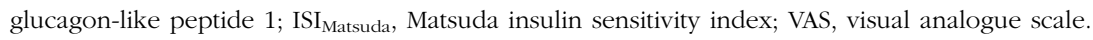

*Corresponding author: J. T. Gonzalez, fax +44 191243 7012, email javier.gonzalez@northumbria.ac.uk 
hormonal and metabolic milieu ${ }^{(9)}$, which underscores the importance of determining these changes concomitant with measuring energy balance. Exercise training improves glucose tolerance $^{(5)}$, yet acute exercise effects are less lucid ${ }^{(10-13)}$. Muscle glucose uptake is increased after exercise ${ }^{(14)}$, as assessed in rat hindlimb muscle. However, both this method and the most commonly used technique for assessing insulin sensitivity in human subjects (the euglycaemic-hyperinsulinaemic clamp) possess some caveats. First, they ignore the gastrointestinal response to food ingestion. Direct contact of nutrients with L-cells in the intestine stimulates secretion of glucagon-like peptide 1 (GLP-1), which potentiates insulin secretion and sensitivity and reduces food intake ${ }^{(9)}$. GLP-1 exists in two active forms; in human subjects, the primary circulating form is $\mathrm{GLP}-1_{7-36}{ }^{(9)}$. Acute exercise has been shown to increase GLP-1 concentrations in the fed state ${ }^{(15)}$. Therefore, GLP-1 may be an important mediator in the acute regulation of energy homeostasis regarding breakfast consumption and exercise.

Second, provision of nutrients other than glucose can influence glucose tolerance and insulin sensitivity. Protein, for example, stimulates insulin and/or incretin hormone secretion $^{(16)}$. Flavoured milk providing mixed macronutrients is an increasingly consumed post-exercise drink due to its recovery-enhancing potential ${ }^{(17)}$. Therefore, assessing the whole-body metabolic and endocrine response to an orally ingested mixed-nutrient load provides more applicable findings to regular exercisers. Acute exercise can transiently suppress hunger ${ }^{(15,18)}$, possibly via changes in appetite-related hormones ${ }^{(15,18,19)}$. Subsequent relative energy intake is usually also reduced ${ }^{(18,19)}$. The influence of nutritional status on appetite regulation and energy intake following exercise is not entirely understood. Of the studies investigating appetite responses to fasted $v$. fed exercise, one used a high-fat $(70 \%)$ meal ${ }^{(20)}$, which is not representative of a typical breakfast, and another compared meal-exercise sequence rather than omission of breakfast per se ${ }^{(21)}$.

Accordingly, the aim of the present study was to explore the interaction of breakfast consumption and exercise on the metabolic, endocrine and appetite responses to a commonly consumed post-exercise drink, and to assess subsequent energy intake and macronutrient balance in physically active males.

\section{Materials and methods}

\section{Participants}

A group of twelve healthy males was recruited from the student and staff population at Northumbria University between December 2010 and April 2011. All participants gave informed written consent and completed the entire study. Participants who self-reported as physically inactive, defined by less than $30 \mathrm{~min}$ of moderate activity, five times per week by the International Physical Activity Questionnaire ${ }^{(22)}$; restrained eaters, defined by a score of $>11$ on the Three Factor Eating Questionnaire ${ }^{(23)}$; or those with any metabolic disorders or on medications were omitted.
The protocol was approved by the School of Life Sciences Ethics Committee at Northumbria University.

\section{Preliminary measurements}

Participants undertook preliminary tests to establish: (1) the relationship between $\mathrm{O}_{2}$ uptake and running speed on a flat treadmill (Woodway ELG, Woodway) using a four-stage, 16 min test; (2) their $\mathrm{V}_{\text {O2peak }}$ using an incremental treadmill test, whereby the gradient was increased by $1 \% / \mathrm{min}$ to exhaustion, as described previously in detail ${ }^{(24)}$. The duration of the exercise period in the main trials was calculated from submaximal $\mathrm{O}_{2}$ uptake and $\mathrm{CO}_{2}$ values in order to expend $2.9 \mathrm{MJ}(693 \mathrm{kcal})$ of energy whilst running at a speed estimated to elicit $60 \% \mathrm{~V}_{\text {O2peak. This value was chosen to equate to }}$ approximately $1 \mathrm{~h}$ on average, whilst maintaining similar energy expenditure across participants. On the same day, participants were familiarised with the visual analogue scales (VAS) to assess subjective appetite sensations in main trials, and it was verbally confirmed that participants did not have any particular disliking of foods contained in the test meals.

\section{Experimental design}

All participants completed four trials in a randomised (performed by J. T. G with Research Randomizer version 3.0; http://www.randomizer.org/), crossover design separated by $\geq 7 \mathrm{~d}$ comprising a continued overnight fast followed by: (1) rest without breakfast (FR); (2) exercise without breakfast (FE); (3) breakfast consumption (1859 kJ) followed by rest (BR); and (4) breakfast consumption followed by exercise (BE). By necessity of the design (food intake and exercise), the intervention was not blinded. All trials were performed under similar laboratory conditions (ambient temperature, humidity and pressure; all $P>0.05$; data not shown). Food and fluid diaries were kept for the day preceding the first trial and participants were instructed to replicate this for all subsequent trials. Alcohol, caffeine and vigorous activity were prohibited for $24 \mathrm{~h}$ prior to trials.

On trial days, participants arrived in the laboratory at 07.30 hours after a $10-14 \mathrm{~h}$ fast and a cannula was inserted into an antecubital vein for blood sampling. After baseline samples of expired gas and VAS were taken, in breakfast trials (BE and BR), participants consumed a porridge breakfast. In fasting trials (FE and FR), participants were permitted to consume water only, which was consumed ad libitum on the first exercise and non-exercise trials, and water consumption was replicated for the following exercise and non-exercise trials (Fig. 1). Following $120 \mathrm{~min}$ of rest, during exercise trials (BE and FE), participants ran on a treadmill at 61.1 (SEM 0.6) $\%$ $\mathrm{V}_{\text {O2peak }}$ for 59 (SEM 2) min based on the a priori estimated energy expenditure. Treadmill speed was adjusted accordingly on the first trial to obtain the appropriate $\mathrm{V}_{\mathrm{O} 2}$. Changes in speed were noted for duplication in subsequent exercise trials. In resting trials (BR and FR), participants rested for the equivalent amount of time as the exercise trials.

Within 20 min of exercise termination, participants ingested a chocolate milk test drink. Following a 90 min postprandial 


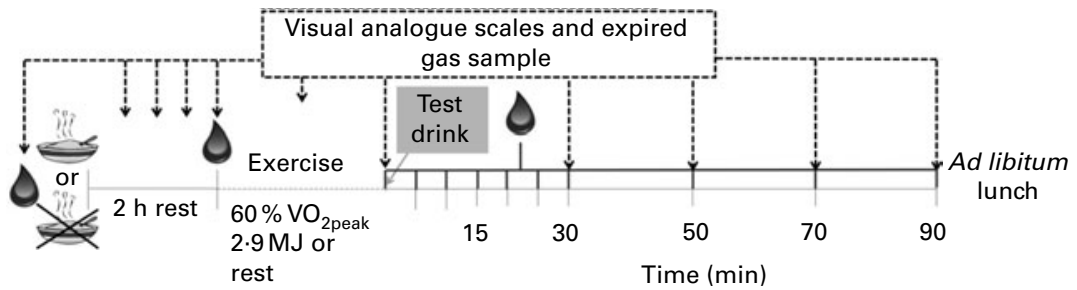

Fig. 1. Schematic representation of trials. ¿, Breakfast consumption; $₫$, blood sample.

period, a homogeneous ad libitum test lunch was provided. Participants were provided with an initial $430 \mathrm{~g}(3694 \mathrm{~kJ}$; $882 \mathrm{kcal}$ ) portion of the test meal, which was replaced upon completion. The test meal was terminated when the participant instructed that they felt 'comfortably full'. Participants were constantly reminded to follow this instruction and were always presented with fresh, warmed portions before participant-induced termination to ensure that the end of a portion was not the reason for meal termination. Remaining food was then removed and weighed out of the sight of the participants to determine energy intake.

\section{Anthropometric measurements}

Body mass was determined to the nearest $0 \cdot 1 \mathrm{~kg}$ using balance scales (Seca) upon arrival at the laboratory, immediately prior to and following exercise, where participants wore only light clothing. Height was measured to the nearest $0.1 \mathrm{~cm}$ using a stadiometer (Seca).

\section{Test meals}

The breakfast consisted of $72 \mathrm{~g}$ oats (Oatso Simple Golden Syrup, Quaker Oats) and $360 \mathrm{ml}$ semi-skimmed milk (Tesco) and provided $1859 \mathrm{~kJ}$ of energy ( $444 \mathrm{kcal} ; 17 \%$ protein, $60 \%$ carbohydrate and $23 \%$ fat). The test drink was $500 \mathrm{ml}$ of chocolate milk (Yazoo, Campina Limited) and contained $1500 \mathrm{~kJ}$ of energy $(358 ; 18 \%$ protein, $63 \%$ carbohydrate and $19 \%$ fat). The test lunch comprised pasta (Tesco), tomato sauce (Tesco), cheddar cheese (Tesco) and olive oil (Tesco) and provided $859 \mathrm{~kJ}$ of energy per $100 \mathrm{~g}$ of food $(205 \mathrm{kcal}$; $14 \%$ protein, $52 \%$ carbohydrate and $34 \%$ fat).

\section{Blood sampling and analysis}

Blood samples, $10 \mathrm{ml}$, were collected at baseline, immediately prior to and following exercise (or the equivalent points in resting trials) at 15, 30, 50, 70 and 90 min following consumption of the test drink (immediately prior to the test meal). All samples were obtained whilst participants were seated upright to control for postural changes in plasma volume. Additional $5 \mathrm{ml}$ samples were collected at 5, 10, 20 and $25 \mathrm{~min}$ following test drink ingestion, where blood glucose was determined immediately by a glucose analyser (Biosen C_line, EKF Diagnostics). From the $10 \mathrm{ml}$ samples, a $20 \mu \mathrm{l}$ capillary tube was filled with whole blood to determine blood glucose concentrations, $4 \mathrm{ml}$ was dispensed into an EDTA vacutainer containing $100 \mu \mathrm{l}$ aprotinin and immediately centrifuged at $3000 \mathrm{rpm}$ at $4^{\circ} \mathrm{C}$ for $10 \mathrm{~min}$. Plasma was stored for later determination of $\mathrm{GLP}_{-1-36}$ using an immunoassay (Phoenix Pharmaceuticals, Inc.). Remaining whole blood from $10 \mathrm{ml}$ samples was allowed to stand for $30 \mathrm{~min}$ in a non-anticoagulant tube before being centrifuged at $3000 \mathrm{rpm}$ at $4^{\circ} \mathrm{C}$ for $10 \mathrm{~min}$. Aliquots of serum were then stored for later determination of NEFA (WAKO Diagnostics) and insulin (DIAsource ImmunoAssays S.A.) concentrations in duplicate. All plasma/serum samples were stored at $-80^{\circ} \mathrm{C}$. The intra-assay $\mathrm{CV}$ were $5 \cdot 6$ and $7 \cdot 2 \%$ for NEFA and insulin, respectively. Inter-assay CV were $8.1,3.6$ and $18.5 \%$ for NEFA, insulin and GLP-1 $1_{7-36}$, respectively. In order to reduce the inter-assay variation, samples from each participant were analysed during the same run where possible. It was decided that it was unnecessary to adjust analyte concentrations to account for plasma volume changes, as exercise of a similar and greater intensity and duration does not result in changes in plasma volume ${ }^{(15,25)}$.

\section{Energy expenditure and substrate oxidation}

Expired gas samples were collected using an online gas analysis system (Metalyzer 3B, Cortex) calibrated using gases of known concentrations and a 31 syringe. Participants wore a facemask and after a 2 min stabilisation phase, 5 min samples were obtained and averaged at baseline, at every $30 \mathrm{~min}$ after breakfast consumption (or equivalent time in breakfast omission trials) and at 5, 15, 30, 50, 70 and 90 min following consumption of the test drink. Expired gas was continuously sampled throughout the exercise and averaged over each $5 \mathrm{~min}$ period, ignoring the first $5 \mathrm{~min}$ to allow for steady-state values.

Substrate metabolism was calculated, assuming negligible protein oxidation, with $\mathrm{V}_{\mathrm{O} 2}$ and $\mathrm{CO}_{2}$ production values using stoichiometric equations and was adjusted during exercise to account for the contribution of glycogen to metabolism ${ }^{(26)}$ :

Rate of fat oxidation at rest and during exercise $(\mathrm{g} / \mathrm{min})$
$\quad=\left(1.695 \times \mathrm{V}_{\mathrm{O} 2}\right)-\left(1.701 \times \mathrm{V}_{\mathrm{CO} 2}\right)$

$$
\text { Rate of carbohydrate oxidation at rest }(\mathrm{g} / \mathrm{min})
$$

$$
=\left(4.585 \times \mathrm{V}_{\mathrm{CO} 2}\right)-\left(3.226 \times \mathrm{V}_{\mathrm{O} 2}\right) \text {. }
$$

Rate of carbohydrate oxidation during exercise $(\mathrm{g} / \mathrm{min})$

$$
=\left(4.210 \times \mathrm{V}_{\mathrm{CO} 2}\right)-\left(2.962 \times \mathrm{V}_{\mathrm{O} 2}\right) \text {. }
$$

$\mathrm{V}_{\mathrm{O} 2}$ and $\mathrm{V}_{\mathrm{CO} 2}$ are measured in litres/min.

Energy expenditure was calculated based on fat, glucose and glycogen concentrations providing 40.81, 15.64 and $17 \cdot 36 \mathrm{~kJ} / \mathrm{g}$ of energy, respectively. At rest, calculations were based on glucose providing all of the carbohydrate for metabolism, whereas during moderate-intensity exercise,

\footnotetext{
containing $100 \mu$ aprotinin and immediately centrifuged
} 
carbohydrate oxidation is met by both glucose and glycogen providing a 20 and $80 \%$ contribution, respectively ${ }^{(26)}$.

\section{Subjective ratings}

Paper-based $100 \mathrm{~mm}$ VAS were completed at baseline, prior to and immediately following breakfast and at every $30 \mathrm{~min}$ thereafter until exercise (or equivalent time points in breakfast omission trials); further, VAS were completed immediately following exercise and after test drink consumption and at $30 \mathrm{~min}$ intervals thereafter. Final VAS were completed following termination of the test meal. Questions asked were used to determine hunger, fullness, satisfaction and prospective food consumption. An overall appetite score was calculated using the following formula, as previously used ${ }^{(27)}$ :

$$
\begin{aligned}
& \text { Overall appetite } \\
& \begin{array}{l}
=(\text { hunger }+ \text { prospective food consumption } \\
\quad+(100-\text { fullness })+(100-\text { satisfaction })) / 4
\end{array}
\end{aligned}
$$

\section{Statistical analysis}

Due to difficulties associated with blood collection, data for GLP- $1_{7-36}$ are presented from ten participants and, for all other blood analytes, from eleven participants. After the consumption of the test drink, glucose, insulin, GLP-1 ${ }_{7-36}$ and NEFA concentrations and appetite sensations were converted into AUC using the trapezoidal rule. Indices of insulin secretion and sensitivity, post-test drink serum insulin AUC to blood glucose $\mathrm{AUC}$ ratio $\left(\mathrm{AUC}_{\mathrm{INS} / \mathrm{GLU}}\right)$ and Matsuda insulin sensitivity index (ISI $\mathrm{Iatsuda}_{\text {a }}$ ) were calculated as described previously $^{(28,29)}$. Unless otherwise stated, all data are presented as mean values with their standard errors. One-way, repeated measures ANOVA was used to determine differences at baseline, between all AUC values and total fat and carbohydrate oxidation and energy expenditure between trials. Two-way repeated measures ANOVA (trial $\times$ time) was used to detect differences for all variables, and following a significant interaction effect, simple main effects analyses were employed. This approach allowed for a comparison between the four conditions (FR, FE, BR and $\mathrm{BE}$ ) across time to determine the most appropriate diet/exercise strategy. The HolmBonferroni step-wise post hoc test was utilised to determine the location of the variance, and all $P$ values reported have already been adjusted for multiple comparisons. Differences were considered significant at $P<0 \cdot 05$.

\section{Results}

The participants' age, height, body mass, BMI and peak $\mathrm{O}_{2}$ uptake $\left(\mathrm{V}_{\text {O2peak }}\right)$ were $23 \cdot 2(\mathrm{SD} 4 \cdot 3)$ years, $178 \cdot 0(\mathrm{SD} 7 \cdot 0) \mathrm{cm}$, $77 \cdot 2(\mathrm{sD} 5 \cdot 3) \mathrm{kg}, 24.5(\mathrm{sD} 2 \cdot 0) \mathrm{kg} / \mathrm{m}^{2}$ and $53 \cdot 1(\mathrm{sD} 5 \cdot 5) \mathrm{ml} / \mathrm{kg}$ per min, respectively.

\section{Blood glucose}

Blood glucose concentration displayed a trial $\times$ time interaction effect (Fig. 2(A); $P<0 \cdot 001$ ). Breakfast consumption
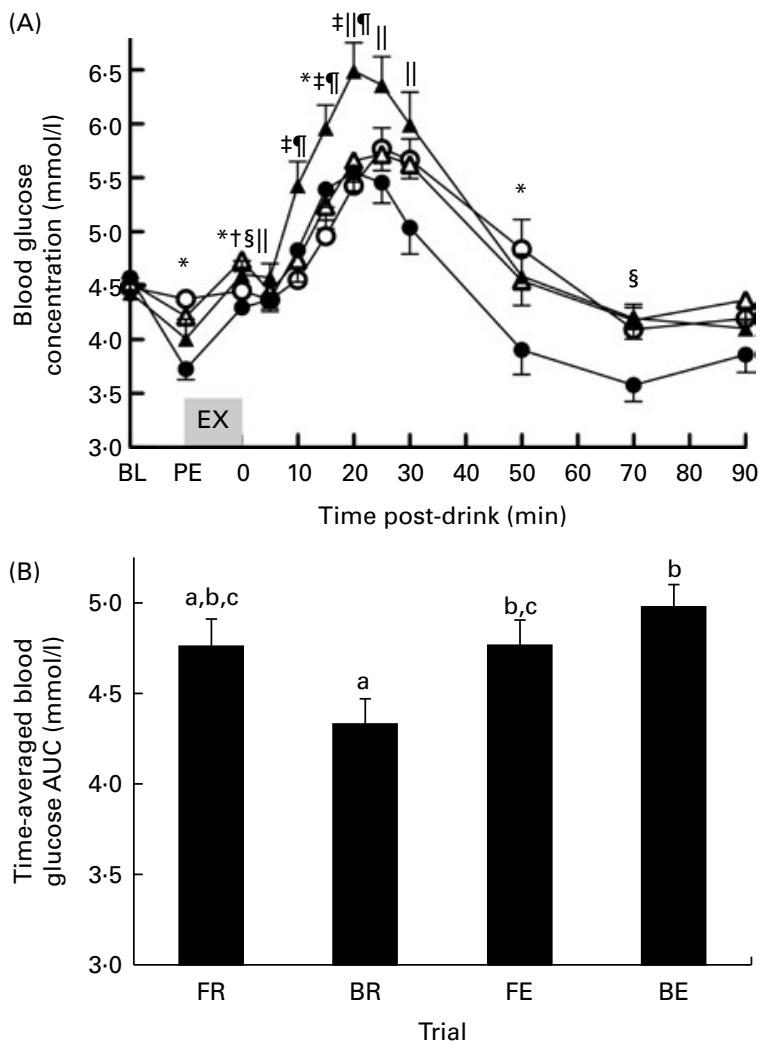

Fig. 2. (A) Blood glucose concentration in response to test drink consumption in the overnight fast followed by rest without breakfast (FR, O), overnight fast followed by breakfast and rest (BR, ๑), overnight fast followed by exercise (EX) without breakfast (FE, $\Delta$ ) and overnight fast followed by breakfast and $\mathrm{EX}(\mathrm{BE}, \boldsymbol{\Delta})$ trials. $\mathrm{BL}$, baseline; PE, pre-EX. Values are means, with their standard errors represented by vertical bars. ${ }^{*}$ Mean value for the FE trial was significantly different from that of $\mathrm{BR}$ trial $(P<0.05)$. † Mean value for the FR trial was significantly different from that of FE trial $(P<0.05)$. ¥Mean value for the FR trial was significantly different from that of $\mathrm{BE}$ trial $(P<0.05)$. $\S$ Mean value for the BR trial was significantly different from that of $\mathrm{FE}$ trial $(P<0.05)$. II Mean value for the BR trial was significantly different from that of $\mathrm{BE}$ trial $(P<0.05)$. I Mean value for the FE trial was significantly different from that of BE trial $(P<0.05)$. (B) Time-averaged blood glucose AUC following test drink consumption. ${ }^{a, b, c}$ Values with unlike letters were significantly different $(P<0.05)$.

reduced time to reach peak blood glucose concentration following test drink ingestion by 10 and $4 \mathrm{~min}$ during rest and exercise trials, respectively $(P=0.012$ and $P=0.02$, respectively). Peak blood glucose concentration was unaffected by breakfast consumption during resting trials (FR: 5.95 (sem 0.20) mmol/l, BR: $5 \cdot 75$ (sem 0.14) $\mathrm{mmol} / \mathrm{l} ; P=0 \cdot 20)$. No difference was observed in peak or in time to peak blood glucose concentrations in FR $v$. FE trials $(P=0.73$ and $P=0 \cdot 28$, respectively). However, in $\mathrm{BE}$, blood glucose concentration reached $6.66(\mathrm{SEM} 0.24) \mathrm{mmol} / \mathrm{l}$, significantly greater than FE $(5.89$ (SEM 0.17$) \mathrm{mmol} / \mathrm{l} ; P=0.06)$ and BR $(P=0.030)$. The difference between the BR and FR trials in AUC for blood glucose approached statistical significance (Fig. 2(B); $P=0.09)$; but it was not significantly different between the FR and FE trials $(P=0.65)$; and was greater in $\mathrm{BE} v$. BR trials $(P=0 \cdot 012)$. 

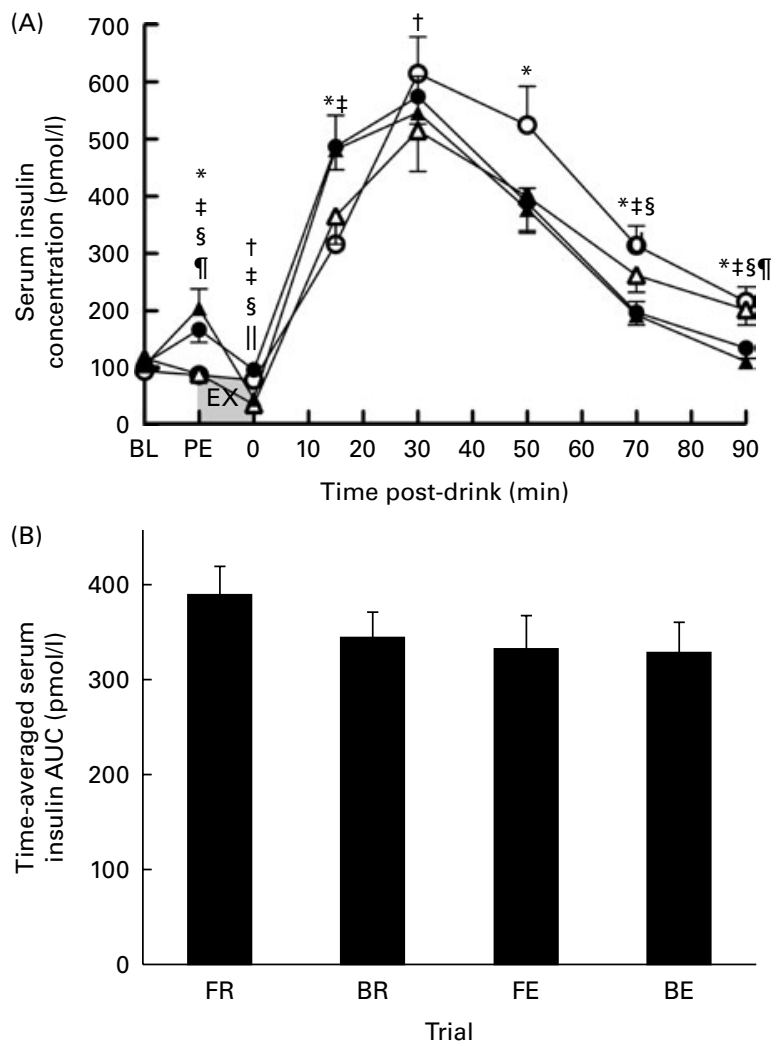

Fig. 3. (A) Serum insulin concentration in response to test drink consumption in the overnight fast followed by rest without breakfast (FR, $O$ ), overnight fast followed by breakfast and rest (BR, $\bullet$ ), overnight fast followed by exercise (EX) without breakfast ( $\mathrm{FE}, \Delta$ ) and overnight fast followed by breakfast and $\mathrm{EX}(\mathrm{BE}, \mathbf{\Lambda})$ trials. BL, baseline; $\mathrm{PE}$, pre-EX. Values are means, with standard errors represented by vertical bars. * Mean value for the FE trial was significantly different from that of $\mathrm{BR}$ trial $(P<0.05)$. † Mean value for the $\mathrm{FR}$ trial was significantly different from that of $\mathrm{FE}$ trial $(P<0.05)$. $¥$ Mean value for the FR trial was significantly different from that of $\mathrm{BE}$ trial $(P<0.05)$. § Mean value for the $\mathrm{BR}$ trial was significantly different from that of $\mathrm{FE}$ trial $(P<0.05)$. $\|$ Mean value for the BR trial was significantly different from that of $B E$ trial $(P<0.05)$. ๆ Mean value for the FE trial was significantly different from that of $\mathrm{BE}$ trial $(P<0.05)$. (B) Time-averaged serum insulin AUC following test-drink consumption.

\section{Serum insulin}

A trial $\times$ time interaction effect was observed for serum insulin concentrations $(P<0 \cdot 001)$, where peak concentrations occurred at 37 (SEM 3) min in the FR trial, and the delay compared with BR (29 (SEM 1) min; $P=0.09$ ) and FE (30 (sem 4) $\mathrm{min} ; P=0 \cdot 10$ ) approached statistical significance. Serum insulin concentrations rose after test drink consumption (Fig. 3(A)) to a similar peak between trials (FR: 682 (SEM 71), BR: 607 (SEM 46), FE: 570 (SEM 72) and BE: 586 (sEM 64) $\mathrm{pmol} / \mathrm{l} ; P=0 \cdot 21)$. The greater AUC for serum insulin in FR $v$. all other trials approached statistical significance (Fig. 3(B); $P=0.07, P=0.12$ and $P=0.09$ for BR, FE and BE, respectively).

\section{Indices of insulin secretion and sensitivity}

The $\mathrm{AUC}_{\text {INS/GLU }}$ was similar between FR and BR trials (82 (SEM 7) and 80 (sem 6) pmol $/ \mathrm{mmol} ; P=0 \cdot 45$ ), but was reduced by exercise compared with the FR trial (FE: 70 (SEM 7) and BE: 67 (sem 6) $\mathrm{pmol} / \mathrm{mmol} ; P=0.03$ and $P=0.04$ for $\mathrm{FE}$ and $\mathrm{BE}$ trials, respectively). ISI $_{\text {Matsuda }}$ was similar between the trials (12 (SEM 4), 12 (SEM 4), 12 (SEM 4) and 13 (SEM5) arbitrary units for FR, BR, FE and BE respectively; all $P>0.05)$.

\section{Serum NEFA}

Test drink consumption transiently suppressed NEFA concentrations and a significant trial $\times$ time interaction effect was observed (Fig. 4(A); $P<0 \cdot 001$ ). The time at which the nadir of NEFA concentrations was reached was delayed in the FR trial (81 (SEM 3) min) compared with all other trials (BR: 65 (SEM 3) min, $P=0.019$; FE: 57 (sem 3) min, $P<0.001$; and BE: 55 (sem 6) min, $P=0.007)$. The AUC for BR was lower than that for FR and BE trials (Fig. 4(B); $P=0 \cdot 019$ and $P=0 \cdot 004$, respectively).

\section{Plasma glucagon-like peptide 17-36 $_{7}$}

There was no trial $\times$ time interaction effect or main effects of trial on GLP-1 $1_{7-36}$ concentrations (Fig. 5(A); both $P>0.05$ ).
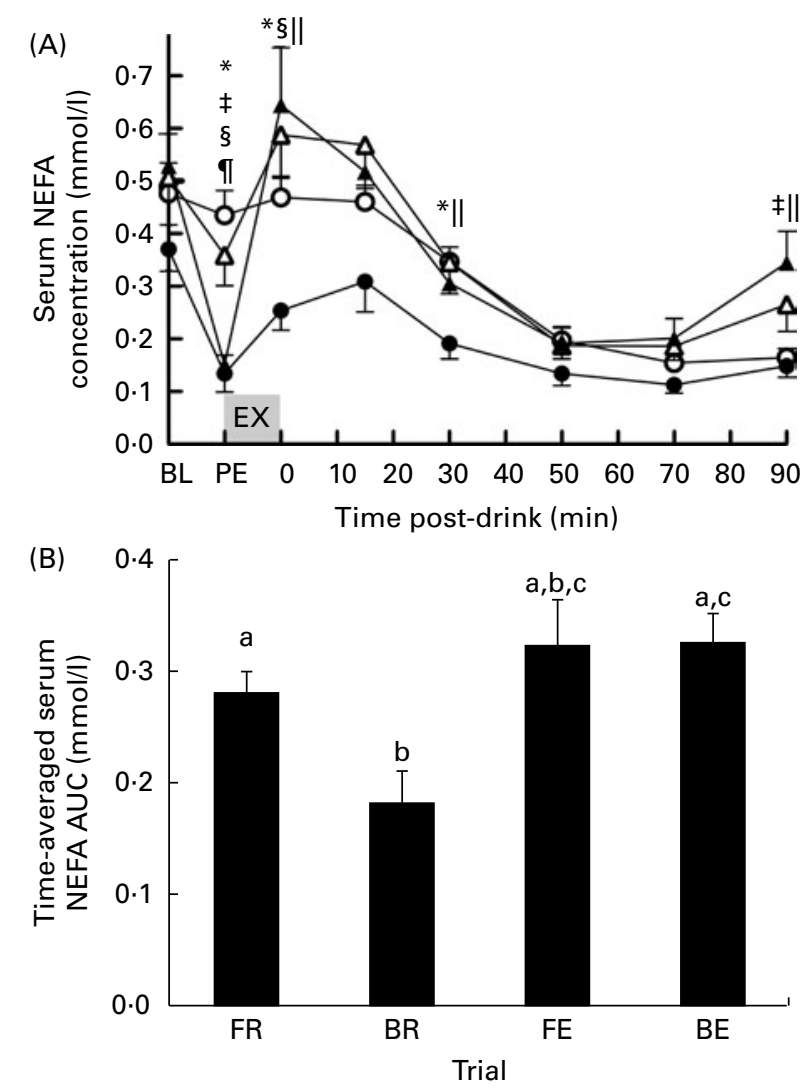

Fig. 4. (A) Serum NEFA concentration in response to test drink consumption in the overnight fast followed by rest without breakfast (FR, O), overnight fast followed by breakfast and rest $(\mathrm{BR}, \bullet)$, overnight fast followed by exercise (EX) without breakfast (FE, $\Delta$ ) and overnight fast followed by breakfast and EX (BE, $\mathbf{\Delta})$ trials. BL, baseline; PE, pre-EX. Values are means, with standard errors represented by vertical bars. ${ }^{*}$ Mean value for the FE trial was significantly different from that of BR trial $(P<0.05)$. † Mean value for the FR trial was significantly different from that of FE trial $(P<0.05)$. $¥$ Mean value for the FR trial was significantly different from that of $\mathrm{BE}$ trial $(P<0.05)$. § Mean value for the BR trial was significantly different from that of FE trial $(P<0.05)$. \| Mean value for the $\mathrm{BR}$ trial was significantly different from that of $\mathrm{BE}$ trial $(P<0.05)$. I Mean value for the $F E$ trial was significantly different from that of $B E$ trial $(P<0.05)$. (B) Time-averaged serum NEFA AUC following test-drink consumption. ${ }^{\mathrm{a}, \mathrm{b}, \mathrm{c}}$ Values with unlike letters were significantly different $(P<0.05)$. 

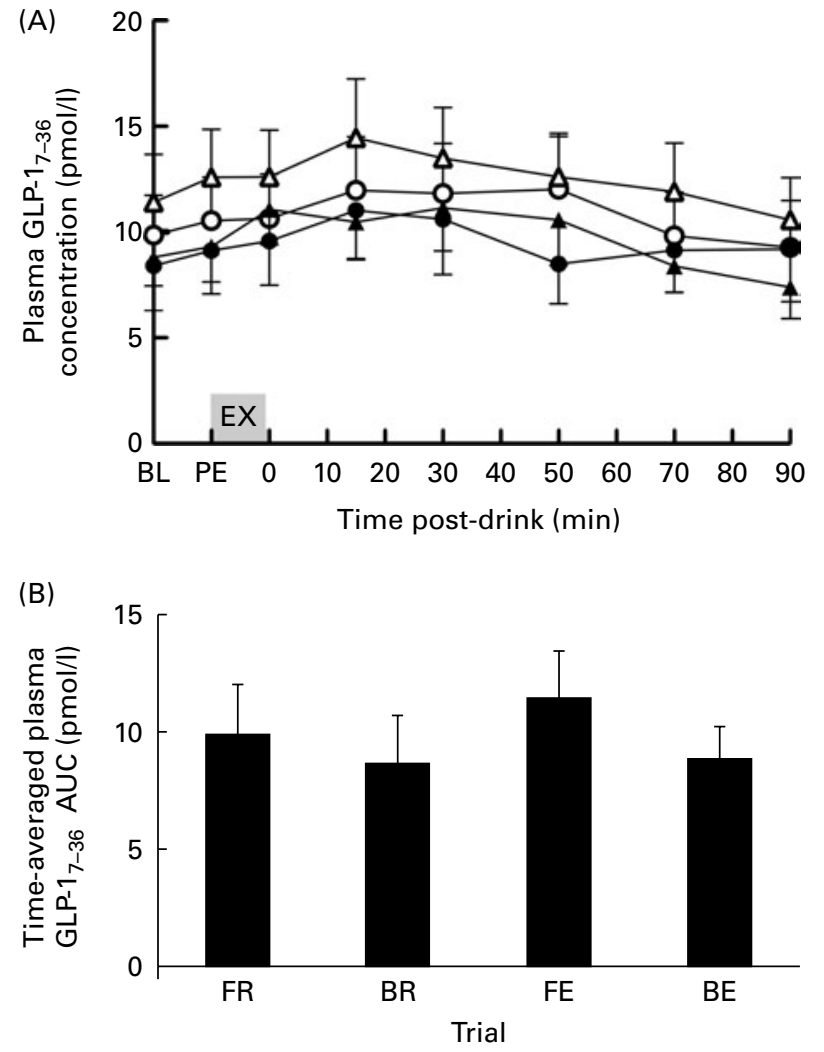

Fig. 5. (A) Plasma glucagon-like peptide- $1_{7-36}\left(\mathrm{GLP}-1_{7-36}\right)$ concentration in response to test drink consumption in the overnight fast followed by rest without breakfast $(\mathrm{FR}, \mathrm{O})$, overnight fast followed by breakfast and rest (BR, @), overnight fast followed by exercise (EX) without breakfast (FE, $\Delta$ ) and overnight fast followed by breakfast and $\mathrm{EX}(\mathrm{BE}, \mathbf{\Delta})$ trials. $\mathrm{BL}$, baseline; $\mathrm{PE}$, pre-EX. (B) Time-averaged GLP-1 $1_{7-36}$ AUC following test drink consumption. Values are means, with standard errors represented by vertical bars.

There was also no difference in AUC (Fig. 5(B)), peak or time to peak GLP- $1_{7-36}$ concentrations $(P=0 \cdot 17, P=0 \cdot 27$ and $P=0 \cdot 45$, respectively).

\section{Energy intake, metabolism and balance}

Energy expenditure, fat oxidation and carbohydrate oxidation did not differ at baseline $(P=0.43, P=0.13$ and $P=0.57$, respectively).

In the breakfast postprandial period, energy expenditure was not significantly different between the trials (Table 1). Less fat and more carbohydrate were utilised during the breakfast postprandial period in the breakfast trials (i.e. BE and BR) $v$. fasting trials (i.e. FE and FR) (Table $1 ; P=0.005$ and $P<0 \cdot 001$, respectively).

The exercise bout lasted for 59 (SEM 2) min and mean $\mathrm{O}_{2}$ uptake was similar between the $\mathrm{FE}$ and $\mathrm{BE}$ trials during this period (2.52 (SEM 0.11) and 2.50 (SEM 0.11) litres/min; $P=0.54$ ). In spite of the equivalent amount of external work performed, exercise increased energy expenditure more during the breakfast trials (3279 (SEM 50) kJ) compared with that during the fasting trials $(2627$ (SEM 43$) \mathrm{kJ} ; P<0 \cdot 01)$. Breakfast consumption reduced the reliance on fat as a substrate and subsequently raised carbohydrate metabolism in the exercise period, an effect which was independent of exercise/rest (Table 1). This resulted in similar carbohydrate balance (intake minus oxidation) post-exercise between $\mathrm{FE}$ and $\mathrm{BE}$, in spite of a large difference in carbohydrate balance prior to exercise (pre-exercise: - 17 (SEM 2) and 43 (SEM 2) g, $P<0.001$; post-exercise: -108 (SEM 7) and -102 (SEM 8) g, $P=0.38$ for $\mathrm{FE}$ and $\mathrm{BE}$ trials, respectively). Following consumption of the test drink, energy expenditure and fat oxidation were greater in both exercise trials compared with rest trials, yet carbohydrate oxidation was similar (Table 1).

There was no detectable difference in ad libitum energy intake at lunch (Fig. 6; $P=0 \cdot 78$ ). Hence, when energy intakes from the breakfast and the test drink are taken into consideration, breakfast trials produced a greater total energy intake (Fig. 6; $P<0.001$ ). The variation in the compensation of energy intake to account for the increase in energy expenditure (energy intake on exercise trials minus energy intake on resting trials) ranged from -1916 to $3749 \mathrm{~kJ}(-458$ to $895 \mathrm{kcal})$ in the fasting trials and from -1447 to $3683 \mathrm{~kJ}$ ( -346 to $880 \mathrm{kcal})$ in the breakfast trials. A total of seven individuals consumed less in the FE $v$. FR trial, four individuals partially compensated for exercise, consuming more in the FE $v$. FR trial, but not enough to overcome the exercise-induced energy expenditure. Only one participant over-compensated for exercise, consuming more than the exercise-induced energy expenditure in the FE $v$. FR trial. In breakfast trials, six individuals consumed less in the BE $v$. BR trial, five partially compensated and only one over-compensated for the exercise-induced energy expenditure. No significant relationship was present between the compensation on fast days and the compensation on breakfast days $(r-0.07, P>0 \cdot 05)$.

Energy balance post-lunch was most positive with BR and least positive with FE trials (Fig. 7). There was no detectable difference in carbohydrate balance when breakfast was omitted $v$. consumed, although the difference at rest approached significance (FR $v$. BR, $P=0 \cdot 06$; FE $v$. BE, $P=0 \cdot 95$; Fig. 7). Yet, fat balance was significantly different between all trials, apart from the $\mathrm{FR} v$. $\mathrm{BE}$ trial, albeit in $\mathrm{BE}$, a reduction which approached statistical significance was observed $(P=0 \cdot 06)$.

\section{Subjective ratings}

Feelings of hunger during the exercise period were suppressed in $\mathrm{FE} v$. FR $(P=0 \cdot 015)$ and $\mathrm{BE} v$. BR trials $(P=0 \cdot 016)$. This was still the case immediately post-exercise in the $\mathrm{FE} v$. FR trial $(P=0 \cdot 002)$, yet, in the $\mathrm{BE} v$. BR trial, there was no detectable difference $(P=0 \cdot 45)$. FE also reduced ratings of prospective consumption during and after exercise $v$. FR ( $P=0.028$ and $P=0.032$, respectively), whereas BE did not significantly affect prospective consumption ratings compared with $\mathrm{BR}(P=0.67$ and $P=0 \cdot 15$, respectively). Overall appetite rating showed similar findings (Fig. 8(A)), where the change from pre- to during the exercise period was significantly different between the FR and the FE trials (2 (SEM 1) $v$. -11 (SEM 4); $P=0.048$ ), but not between the BR and BE trials (6 (SEM 2) v. 0 (SEM 4); $P=0 \cdot 21$ ).

Breakfast did not influence hunger immediately prelunch during exercise trials $(P=0 \cdot 11)$, but did reduce hunger 
in resting trials $(P=0.006)$. The same pattern was observed with prospective consumption (FR $v$. BR: $P=0 \cdot 005$; BR $v$. FE: $P=0 \cdot 005$; FE $v$. BE: $P=0 \cdot 10$ ). However, immediately prior to lunch, overall appetite was suppressed in the BR trial compared with that in both fasting trials (i.e. FE and FR) $(P=0.001$ and $P=0.005$, for rest and exercise, respectively; Fig. 8(B)).

There was no detectable difference in AUC for hunger between exercise and rest $(P=0.47$ and $P=0.71$ for FR $v$. FE and BR $v$. BE trials, respectively). The AUC for overall appetite following consumption of the test drink was greater in the FR trial $v$. the BR trial (Table 2; $P=0 \cdot 006$ ), and this pattern was still apparent, although it was attenuated when exercise was performed (Table 2; $P=0 \cdot 029$ ). Similar patterns were shown for hunger and prospective consumption AUC and mirrored by fullness and satisfaction AUC (Table 2).

\section{Discussion}

The present study attempted to examine the cumulative effects of breakfast consumption and exercise on the metabolic and appetite responses to foods consumed later in the day and on subsequent energy and macronutrient balance. The main findings were that acute breakfast consumption is likely to reduce postprandial glycaemia and insulinaemia at rest. Acute exercise did not affect glucose tolerance when breakfast was omitted, but reduced glucose tolerance when breakfast was consumed; the pertinence of this chronically should be noted with caution, given the benefits of exercise training. Exercise in the fasted state led to a greater transitory reduction in appetite compared with exercise in the fed state. Energy and fat balance were least positive following exercise in the fasted state.

Acute breakfast consumption has been shown to improve glucose tolerance ${ }^{(2)}$. The present findings in physically active males somewhat support the previous data, although the effect may be more trivial in these aerobically fit individuals, with magnitude-based inferences ${ }^{(30)}$ indicating 41 and 59\% likelihoods of beneficial and negligible effects, respectively, on glucose tolerance. This could be due to the fact that subjects in the present study are regular exercisers and therefore displaying better basal glucose tolerance ${ }^{(5)}$. Lower fasting blood glucose concentrations (approximately 4.5 v. $4.8 \mathrm{mmol} / \mathrm{l}$ ) support this proposition. Lower NEFA exposure prior to consumption of the test drink in the BR trial compared with the FR trial is a possible cause of the potential improvement in glucose tolerance, as prolonged NEFA elevations reduce insulin-stimulated glucose disposal by inhibiting insulin signalling $^{(31)}$. The (non-significant) increase in insulinemia and delay in peak insulin concentrations do support this proposition.

Muscle contraction stimulates insulin-independent glucose uptake $^{(14)}$, and thus explains why glucose uptake is augmented following an acute bout of exercise in spite of increased NEFA concentrations, which was observed in the FE and $\mathrm{BE}$ trials. Increased glucose uptake is a well-established observation at the muscle ${ }^{(14)}$ and whole-body level ${ }^{(32)}$. Thus, based on insulin clamp studies, it may seem surprising that there was no difference in glucose tolerance between the fasted rest and exercise trials, but this does, in fact, 


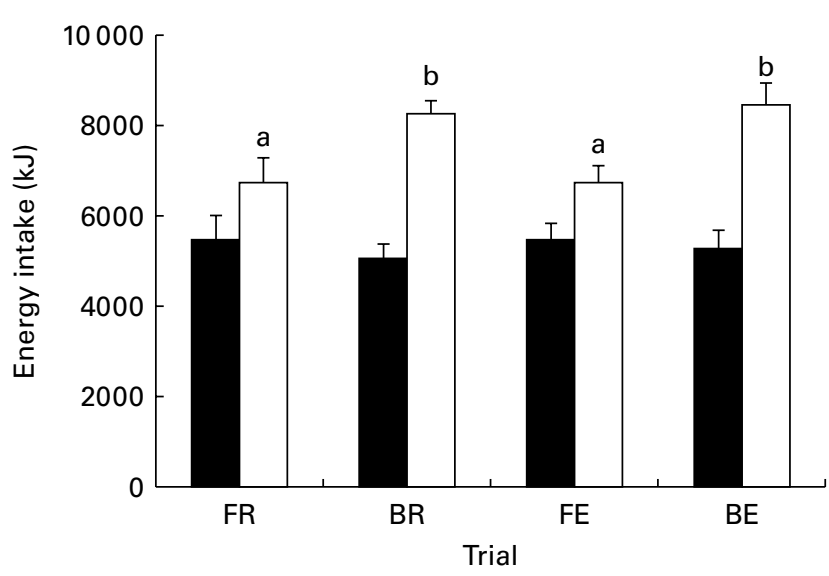

Fig. 6. Energy intake. Energy intake at lunch ( $\square$ ) and throughout the whole trial ( $\square)$. FR, overnight fast followed by rest without breakfast; BR, overnight fast followed by breakfast and rest; FE, overnight fast followed by exercise without breakfast; BE, overnight fast followed by breakfast and exercise. Values are means, with standard errors represented by vertical bars. ${ }^{\mathrm{a}, \mathrm{b}}$ Values with unlike letters were significantly different $(P<0.05)$.

corroborate with studies using oral glucose tolerance tests. Until now, studies in healthy participants have shown either decreases $^{(10,11,33-37)}$ or no difference ${ }^{(12,13,38)}$ in glucose tolerance following acute endurance exercise. In those displaying no difference, the tests were either performed in the fasted state $^{(13,38)}$ or glucose tolerance was assessed more than $2 \mathrm{~h}$ after exercise ${ }^{(12)}$. The present study is the first to demonstrate that when nutrients are ingested immediately post-exercise, the effect on acute postprandial glucose kinetics may depend on the nutritional state (fasted or fed) prior to exercise. It may be the accrual of this acute effect that contributes to the attenuated improvements in glucose tolerance seen during exercise training when carbohydrate availability is high ${ }^{(5)}$.

Regarding the effects of exercise when fasted, endurance exercise increases the rate of appearance of endogenous glucose ${ }^{(37)}$. Therefore, the increase in muscle glucose uptake after exercise ${ }^{(14)}$ (affecting rate of disappearance) could ostensibly be offset by the increase in splanchnic glucose output (affecting rate of appearance) and, hence, result in an increase in flux, but there was no difference in the systemic concentrations of glucose after exercise compared with that after rest when fasted. Future studies are needed to address whether this is indeed the mechanism at play.

Food consumption prior to exercise also increases splanchnic blood flow during exercise ${ }^{(6)}$. As mesenteric blood flow is positively associated with intestinal glucose absorption ${ }^{(39)}$, it can be speculated that the increase in blood flow (from breakfast consumption), combined with increased passive absorption (from exercise), results in the greater peak blood glucose concentration in the $\mathrm{BE}$ trial compared with the $\mathrm{FE}$ trial. However, recent evidence associates the increase in intestinal absorption with reduced gut blood flow occurring during intense exercise and may result in intestinal damage ${ }^{(40)}$, indicating faster entry of glucose into the circulation when gut blood flow is reduced (which occurs when exercising after fasting compared with after feeding ${ }^{(6)}$ ). This adds to the confusion in the previous conjecture, as the putative increase in splanchnic blood flow in BE would result in less intestinal cell damage and reduced passive absorption, leading to a lower blood glucose AUC (assuming that endogenous glucose production and glucose disappearance remain constant, which can be presumed due to similar carbohydrate balance post-exercise and thus similar wholebody glycogen concentrations).

The present study used an exercise intensity that was lower ( $61 \% \mathrm{~V}_{\text {O2peak }} v .70 \%$ of maximum power output) than that of van Wijck et al. ${ }^{(40)}$. At lower intensities $\left(55 \% \mathrm{~V}_{\text {O2peak }}\right)$, the exercise-induced reduction in splanchnic blood flow is abolished $^{(6)}$. This makes it tempting to presume that other factors, such as heat or mechanical stresses or changes in hormone concentrations, contribute to the increase in intestinal glucose absorption following exercise ${ }^{(41)}$. Another factor at play could be reductions in insulin sensitivity of non-exercised (upper limb) muscle following exercise ${ }^{(42)}$. Clearly, this area has great scope for future work, pertinent to the understanding of the impact of food intake and exercise on subsequent whole-body glucose tolerance.

The $\mathrm{AUC}_{\mathrm{INS} / \mathrm{GLU}}$ was lower in both exercise trials compared with the FR trial, whereas ISI $_{\text {Matsuda }}$ was similar between trials, suggesting that postprandial insulin secretion is reduced immediately following exercise, but insulin sensitivity is unaffected $^{(28,29)}$. This strengthens the assumption that the change in glucose kinetics seen in the present study is due to a difference in the glucose rate of appearance.

The finding that GLP- $1_{7-36}$ concentrations were not different between trials is in accordance with the proposition that glucose entered the circulation via passive absorption. Intravenous infusion of glucose mirroring the plasma glucose profile to oral ingestion does not augment GLP-1 concentrations ${ }^{(43)}$. Therefore, as GLP-1 $1_{7-36}$ concentrations were not different between trials, this provides support for elevated glucose appearance from passive absorption, as greater GLP-1 $_{7-36}$ secretion would not occur. GLP-1 ${ }_{7-36}$ is also a potent incretin

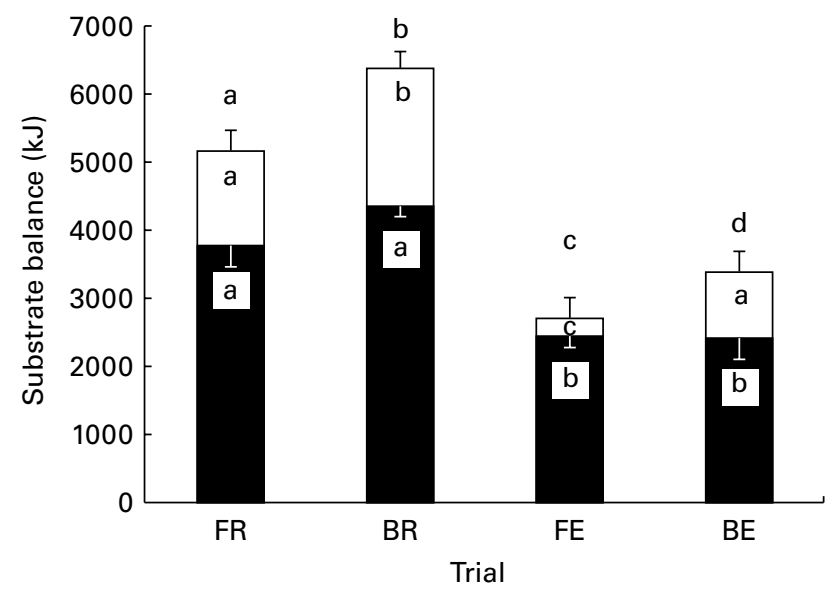

Fig. 7. Substrate balance. Carbohydrate ( $\square$ ), fat $(\square)$ and energy ( $\square$ and $\square$ combined) balance at the end of the trial. FR, overnight fast followed by rest without breakfast; BR, overnight fast followed by breakfast and rest; FE, overnight fast followed by exercise without breakfast; BE, overnight fast followed by breakfast and exercise. Values are means, with standard errors represented by vertical bars. ${ }^{a, b, c, d}$ Values with unlike letters were significantly different $(P<0.05)$. 

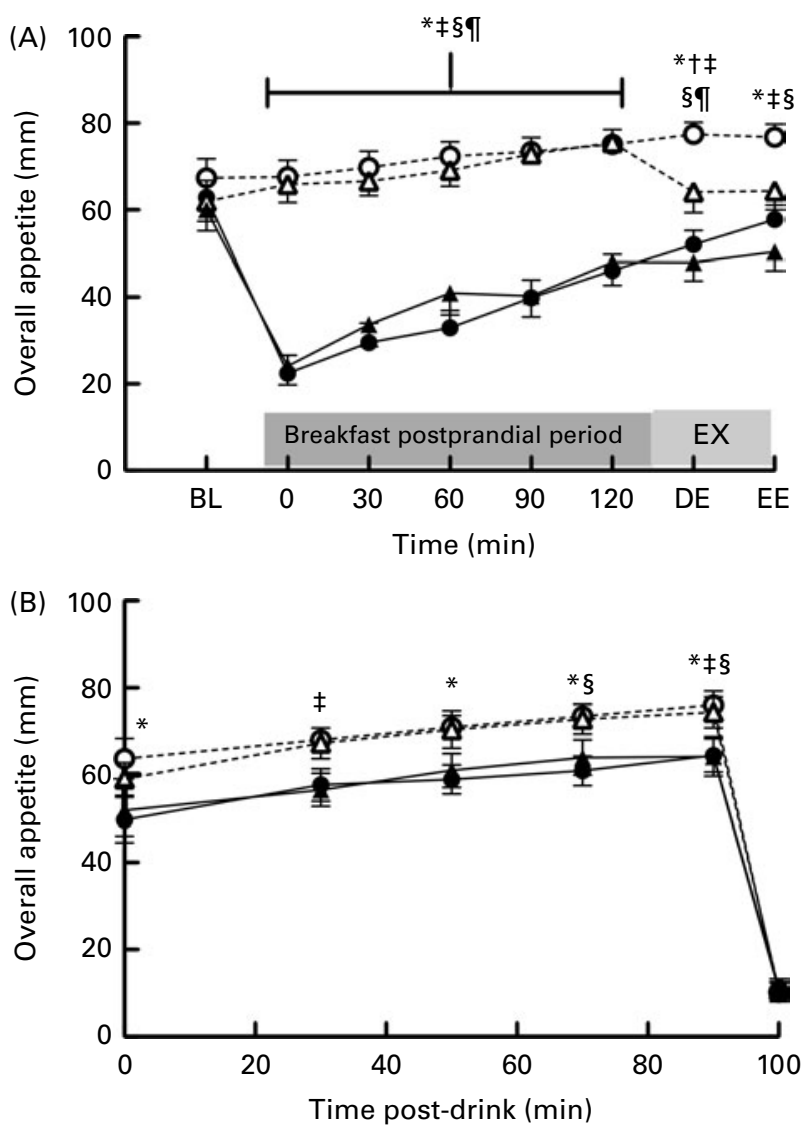

Fig. 8. Overall appetite. Overall appetite sensations during (A) the breakfast postprandial and exercise (EX) periods and (B) following test drink consumption in the overnight fast followed by rest without breakfast (FR, O), overnight fast followed by breakfast and rest (BR, ๑), overnight fast followed by EX without breakfast $(\mathrm{FE}, \Delta)$ and overnight fast followed by breakfast and $\mathrm{EX}$ $(\mathrm{BE}, \mathbf{\Lambda})$ trials. $\mathrm{BL}$, baseline; $\mathrm{DE}$, during $\mathrm{EX} ; \mathrm{EE}$, end of $\mathrm{EX} ; \mathrm{PL}$, post-lunch Values are means, with standard errors represented by vertical bars. * Mean value for the FE trial was significantly different from that of $\mathrm{BR}$ trial $(P<0.05)$. † Mean value for the FR trial was significantly different from that of FE trial $(P<0.05)$. $¥$ Mean value for the $\mathrm{FR}$ trial was significantly different from that of $\mathrm{BE}$ trial $(P<0.05)$. $\S$ Mean value for the BR trial was significantly different from that of $\mathrm{FE}$ trial $(P<0.05)$. ๆ Mean value for the FE trial was significantly different from that of $\mathrm{BE}$ trial $(P<0.05)$.

hormone, stimulating insulin secretion and also suppressing appetite $^{(9)}$. Thus, as GLP-1 $1_{7-36}$ concentration did not differ between trials, it would seem that other factors are playing a role in enhanced insulin action and appetite suppression with breakfast consumption. It should be noted that GLP-1 ${ }_{7-36}$ may interact with neurons expressed locally in L-cells, prior to being rapidly degraded on entry into the circulation, where its clearance can exceed cardiac output by two to three times ${ }^{(44)}$. Hence, GLP-1 ${ }_{7-36}$ can still influence appetite in spite of no detectable rise in its plasma concentrations.

There was evidence of delayed suppression of NEFA following consumption of the test drink in the FR trial compared with the BR trial, suggestive of metabolic inflexibility, again associated with insulin resistance. Exercise uncoupled the link between breakfast, NEFA and insulin concentrations, whereby, in both the FE and BE trials, insulin and NEFA concentrations were similar prior to and following consumption of the test drink. Increased NEFA availability during and following exercise is required to support higher rates of fat oxidation by skeletal muscle as carbohydrate is used to replenish glycogen stores ${ }^{(11)}$. As such, NEFA flux is raised, and, as insulin-resisting effects of NEFA on muscle seem to be time dependent ${ }^{(31)}$, turnover may be more important than NEFA concentrations for insulin sensitivity.

Exercise transiently suppressed hunger and overall appetite. This is a common phenomenon ${ }^{(15,18,45)}$, yet less is known about the effect of nutritional status on the ability of exercise to influence appetite. The present study found that, compared with rest, exercise suppressed hunger and overall appetite to a greater extent when fasted compared with the fed state (approximately $17 v$. 9\%, respectively). Nevertheless, it should be noted that appetite was higher in the fasting state prior to exercise. To our knowledge, this is the first crossover study to demonstrate the effect of exercise in fasted and fed conditions on appetite sensations compared with resting trials in the equivalent nutritional state.

Harmonious with preceding research ${ }^{(15,18)}$, the exerciseinduced suppression of appetite was abolished within $30 \mathrm{~min}$ of exercise termination and appetite was subsequently similar between exercise and rest trials until lunch. Breakfast consumption, however, reduced overall appetite following test drink consumption by approximately 17 and 14\% in the rest and exercise trials, respectively. Despite a $10 \%$ reduction in appetite ratings with breakfast consumption, no detectable difference in energy intake between trials was observed at lunch. This occurred regardless of the additional $1859 \mathrm{~kJ}$ of energy consumed during breakfast and approximately $2423 \mathrm{~kJ}$ of energy expended during exercise. Subsequently, energy intake was higher in breakfast trials. Observational data corroborate the present findings with daily energy intake increase in regular breakfast consumers compared with omitters ${ }^{(1)}$. Yet, when BMI was measured, it was still inversely associated with breakfast consumption ${ }^{(1)}$, suggesting that it may be the increased energy expenditure and the improved metabolic responses to food consumption that result in better weight maintenance.

The outcome that exercise did not influence subsequent energy intake is in accord with most of the prior research in this area, although some have found an increase in immediate energy intake ${ }^{(46)}$. It may be that individual variation exists, whereby some individuals' drive to eat following exercise is dominated by hedonic processes ${ }^{(47)}$. This leads to a divergence between those who compensate for extra energy expenditure by increasing intake and non-compensators, who fail to increase intake in the face of an increase in expenditure. In the present study, the range of compensation for exercise-induced energy expenditure was large $(5665 \mathrm{~kJ}$ of energy separated the individual who over-compensated and the individual who under-compensated the greatest). This variation in the compensation of energy expenditure is likely to account for the variation seen in body fat changes in an exercise intervention (reviewed by Caudwell et al. ${ }^{(48)}$ ). It is interesting to note that there was no significant relationship between the degree of compensation to exercise on fasted trials and breakfast trials, suggesting that those who overcompensate during exercise in one nutritional state (i.e. the 
Table 2. Time-averaged AUC values for subjective appetite responses to consumption of the test drink (Mean values with their standard errors)

\begin{tabular}{|c|c|c|c|c|c|c|c|c|c|c|}
\hline \multirow[b]{2}{*}{ Trial } & \multicolumn{2}{|c|}{ Hunger (mm) } & \multicolumn{2}{|c|}{ Fullness (mm) } & \multicolumn{2}{|c|}{$\begin{array}{l}\text { Satisfaction } \\
(\mathrm{mm})\end{array}$} & \multicolumn{2}{|c|}{$\begin{array}{l}\text { Prospective } \\
\text { consumption } \\
(\mathrm{mm})\end{array}$} & \multicolumn{2}{|c|}{$\begin{array}{l}\text { Overall appe- } \\
\text { tite }(\mathrm{mm})\end{array}$} \\
\hline & Mean & SEM & Mean & SEM & Mean & SEM & Mean & SEM & Mean & SEM \\
\hline $\mathrm{FR}$ & 65 & 4 & 30 & 4 & 27 & 2 & 72 & 3 & 70 & 2 \\
\hline BR & $54^{\star}$ & 4 & 40 & 4 & $40^{*}$ & 3 & $58^{*}$ & 4 & $58^{\star}$ & 3 \\
\hline $\mathrm{FE}$ & 63 & 3 & $28 \dagger$ & 4 & $29 \dagger$ & 3 & $68 \dagger$ & 4 & $67 \dagger$ & 3 \\
\hline $\mathrm{BE}$ & 55 & 4 & 40 & 4 & $40^{*} \ddagger$ & 3 & $62^{*}$ & 4 & $59^{*} \ddagger$ & 4 \\
\hline
\end{tabular}

FR, overnight fast followed by rest without breakfast; BR, overnight fast followed by breakfast and rest; FE, overnight fast followed by exercise without breakfast; BE, overnight fast followed by breakfast and exercise. * Mean value was significantly different from FR $(P<0.05)$.

† Mean value was significantly different from BR $(P<0.05)$. $\ddagger$ Mean value was significantly different from FE $(P<0.05)$.

fasted/fed state) may not over-compensate in the opposing circumstance. Another possibility is that exercise energy expenditure is gradually compensated for by energy intake, which is likely to require a period of several weeks, and even then is not likely to be fully compensated for ${ }^{(49)}$.

The higher total energy intake with breakfast trials and the exercise-induced energy expenditure led to energy balance being most positive in the BR trial and least positive in the FE trial. BE resulted in an approximately $1110 \mathrm{~kJ}$ reduction in energy balance compared with FR. When taken in concert with the similar appetite sensations to resting trials, exercise may provide a more attractive option for restricting energy availability compared with omitting breakfast. Interestingly, in spite of differing quantities of carbohydrate and fat oxidised with all trials, carbohydrate balance was remarkably similar between the FE and BE trials, whereas fat balance was 3-fold more positive in the BE trial. This may not be as clear at rest, as the difference between the FR and BR trials in carbohydrate balance did approach a statistically significant difference (Fig. 7), but was higher than that in exercise trials. At least in the short term, the regulation of carbohydrate stores is more tightly regulated than fat stores ${ }^{(19)}$. The findings of the present study add that consumption/omission of breakfast will not alter carbohydrate balance, whereas exercise can reduce carbohydrate balance.

The increased energy expenditure observed during exercise with breakfast consumption was provided by a higher rate of carbohydrate oxidation, this has previously been reported ${ }^{(50-52)}$ and may be magnified during running due to the weight-bearing component ${ }^{(53)}$. The relevance of this with respect to energy balance was, however, trivial, as energy balance was lower in the FE trial compared with the BE trial.

This controlled experimental study involved the provision of a popular breakfast food consumed prior to a bout of exercise or rest in physically active males, with a structure similar to the eating patterns in Western society. It could be viewed that a caveat with the present study is that the participants were physically active and that a sedentary population would benefit more from exercise/diet-induced improvements in metabolism and appetite. However, those who regularly exercise still utilise energy/carbohydrate restriction in order to regulate body composition ${ }^{(4)}$. Therefore, the results are pertinent to these populations; yet, it would undoubtedly be of virtue to investigate these responses in other populations (females, sedentary and obese) to extrapolate findings to a wider population. Moreover, future work should examine whether there is a difference in energy intake in subsequently consumed meals over a longer duration.

It is also of merit to recognise that the environmental conditions were similar between trials, which is important due to the potential effect of temperature on appetite and energy intake ${ }^{(46)}$.

The findings of the present investigation suggest that in an acute setting, energy intake from breakfast and energy expenditure from exercise are not compensated for at lunch. Consequently, energy balance was most positive following breakfast and rest and least positive following breakfast omission and exercise. When exercise is performed, it may be more pertinent to omit breakfast if a negative fat balance is desirable, although the findings of the present study are unable to predict the longer-term outcomes of energy and fat balance due to the single-meal design, and as such this conclusion should be interpreted with caution.

The present study aimed to explore the effect of breakfast and exercise on the metabolic and appetite responses to subsequent food consumption. The findings indicate that breakfast ingestion may improve the metabolic and appetite responses to subsequently consumed foods when sedentary. When breakfast is consumed, subsequent postprandial glycaemia is higher following exercise, yet care should be taken during the interpretation for chronic effects, as exercise training almost always confers a benefit for glucose tolerance and insulin sensitivity. Exercise also resulted in an ephemeral reduction in appetite, which is greater when performed fasted.

\section{Acknowledgements}

We gratefully thank the volunteers for their participation and A. Wilde for technical assistance. This project received no external funding. J. T. G. and E. J. S. designed the study, J. T. G. and R. C. V. performed the data collection and all authors contributed to data analysis and interpretation and writing of the manuscript. The authors declare no conflicts of interest. 


\section{References}

1. Cho S, Dietrich M, Brown CJ, et al. (2003) The effect of breakfast type on total daily energy intake and body mass index: results from the Third National Health and Nutrition Examination Survey (NHANES III). J Am Coll Nutr 22, 296-302.

2. Astbury NM, Taylor MA \& Macdonald IA (2011) Breakfast consumption affects appetite, energy intake, and the metabolic and endocrine responses to foods consumed later in the day in male habitual breakfast eaters. J Nutr 141, $1381-1389$

3. La Bounty PM, Campbell BI, Wilson J, et al. (2011) International Society of Sports Nutrition position stand: meal frequency. J Int Soc Sports Nutr 8, 4.

4. Morton JP, Robertson C, Sutton L, et al. (2010) Making the weight: a case study from professional boxing. Int J Sport Nutr Exerc Metab 20, 80-85.

5. Van Proeyen K, Szlufcik K, Nielens H, et al. (2010) Training in the fasted state improves glucose tolerance during fat-rich diet. $J$ Physiol 588, 4289-4302.

6. Enevoldsen LH, Simonsen L, Macdonald IA, et al. (2004) The combined effects of exercise and food intake on adipose tissue and splanchnic metabolism. J Physiol 561, 871-882.

7. Stannard SR, Buckley AJ, Edge JA, et al. (2010) Adaptations to skeletal muscle with endurance exercise training in the acutely fed versus overnight-fasted state. J Sci Med Sport 13, 465-469.

8. Nybo L, Pedersen K, Christensen B, et al. (2009) Impact of carbohydrate supplementation during endurance training on glycogen storage and performance. Acta Physiol (Oxf) 197, 117-127.

9. Suzuki K, Simpson KA, Minnion JS, et al. (2010) The role of gut hormones and the hypothalamus in appetite regulation. Endocr J 57, 359-372.

10. Folch N, Peronnet F, Massicotte D, et al. (2001) Metabolic response to small and large ${ }^{13} \mathrm{C}$-labelled pasta meals following rest or exercise in man. Br J Nutr 85, 671-680.

11. Folch N, Peronnet F, Massicotte D, et al. (2003) Metabolic response to a large starch meal after rest and exercise: comparison between men and women. Eur J Clin Nutr 57, $1107-1115$.

12. Venables MC, Shaw CS, Jeukendrup AE, et al. (2007) Effect of acute exercise on glucose tolerance following post-exercise feeding. Eur J Appl Physiol 100, 711-717.

13. Long W 3rd, Wells K, Englert V, et al. (2008) Does prior acute exercise affect postexercise substrate oxidation in response to a high carbohydrate meal? Nutr Metab (Lond) 5, 2.

14. Goodyear LJ, King PA, Hirshman MF, et al. (1990) Contractile activity increases plasma membrane glucose transporters in absence of insulin. Am J Physiol 258, E667-E672.

15. Martins C, Morgan LM, Bloom SR, et al. (2007) Effects of exercise on gut peptides, energy intake and appetite. J Endocrinol 193, 251-258.

16. Frid AH, Nilsson M, Holst JJ, et al. (2005) Effect of whey on blood glucose and insulin responses to composite breakfast and lunch meals in type 2 diabetic subjects. Am J Clin Nutr 82, 69-75.

17. Thomas K, Morris P \& Stevenson E (2009) Improved endurance capacity following chocolate milk consumption compared with 2 commercially available sport drinks. Appl Physiol Nutr Metab 34, 78-82.

18. King JA, Miyashita M, Wasse LK, et al. (2010) Influence of prolonged treadmill running on appetite, energy intake and circulating concentrations of acylated ghrelin. Appetite 54, 492-498.
19. Burton FL, Malkova D, Caslake MJ, et al. (2010) Substrate metabolism, appetite and feeding behaviour under low and high energy turnover conditions in overweight women. Br J Nutr 104, 1249-1259.

20. Cheng MH, Bushnell D, Cannon DT, et al. (2009) Appetite regulation via exercise prior or subsequent to high-fat meal consumption. Appetite 52, 193-198.

21. Borer KT, Wuorinen E, Chao C, et al. (2005) Exercise energy expenditure is not consciously detected due to oro-gastric, not metabolic, basis of hunger sensation. Appetite $\mathbf{4 5}$, 177-181.

22. Craig CL, Marshall AL, Sjostrom M, et al. (2003) International physical activity questionnaire: 12-country reliability and validity. Med Sci Sports Exerc 35, 1381-1395.

23. Stunkard AJ \& Messick S (1985) The three-factor eating questionnaire to measure dietary restraint, disinhibition and hunger. J Psychosom Res 29, 71-83.

24. Williams C, Nute MG, Broadbank L, et al. (1990) Influence of fluid intake on endurance running performance. A comparison between water, glucose and fructose solutions. Eur J Appl Physiol Occup Physiol 60, 112-119.

25. Burns SF, Broom DR, Miyashita M, et al. (2007) A single session of treadmill running has no effect on plasma total ghrelin concentrations. J Sports Sci 25, 635-642.

26. Jeukendrup AE \& Wallis GA (2005) Measurement of substrate oxidation during exercise by means of gas exchange measurements. Int J Sports Med 26, Suppl. 1, S28-S37.

27. Anderson GH, Catherine NL, Woodend DM, et al. (2002) Inverse association between the effect of carbohydrates on blood glucose and subsequent short-term food intake in young men. Am J Clin Nutr 76, 1023-1030.

28. Retnakaran R, Shen S, Hanley AJ, et al. (2008) Hyperbolic relationship between insulin secretion and sensitivity on oral glucose tolerance test. Obesity 16, 1901-1907.

29. Matsuda M \& DeFronzo RA (1999) Insulin sensitivity indices obtained from oral glucose tolerance testing: comparison with the euglycemic insulin clamp. Diabetes Care 22, $1462-1470$.

30. Batterham AM \& Hopkins WG (2006) Making meaningful inferences about magnitudes. Int J Sports Physiol Perform 1, 50-57.

31. Hirabara SM, Silveira LR, Abdulkader F, et al. (2007) Timedependent effects of fatty acids on skeletal muscle metabolism. J Cell Physiol 210, 7-15.

32. Mikines KJ, Sonne B, Farrell PA, et al. (1988) Effect of physical exercise on sensitivity and responsiveness to insulin in humans. Am J Physiol 254, E248-E259.

33. O'Connor AM, Pola S, Ward BM, et al. (2006) The gastroenteroinsular response to glucose ingestion during postexercise recovery. Am J Physiol Endocrinol Metab 290, E1155-E1161.

34. King DS, Baldus PJ, Sharp RL, et al. (1995) Time course for exercise-induced alterations in insulin action and glucose tolerance in middle-aged people. J Appl Physiol $\mathbf{7 8}, 17-22$

35. Krzentowski G, Pirnay F, Luyckx AS, et al. (1982) Metabolic adaptations in post-exercise recovery. Clin Physiol 2, 277-288.

36. Pestell RG, Ward GM, Galvin P, et al. (1993) Impaired glucose tolerance after endurance exercise is associated with reduced insulin secretion rather than altered insulin sensitivity. Metabolism 42, 277-282.

37. Rose AJ, Howlett K, King DS, et al. (2001) Effect of prior exercise on glucose metabolism in trained men. Am J Physiol Endocrinol Metab 281, E766-E771. 
38. Englert V, Wells K, Long W, et al. (2006) Effect of acute prior exercise on glycemic and insulinemic indices. $J \mathrm{Am}$ Coll Nutr 25, 195-202.

39. Williams JH Jr, Mager M \& Jacobson ED (1964) Relationship of mesenteric blood flow to intestinal absorption of carbohydrates. J Lab Clin Med 63, 853-863.

40. van Wijck K, Lenaerts K, van Loon LJ, et al. (2011) Exerciseinduced splanchnic hypoperfusion results in gut dysfunction in healthy men. PLoS One 6, e22366.

41. Lambert GP (2009) Stress-induced gastrointestinal barrier dysfunction and its inflammatory effects. J Anim Sci 87, E101-E108.

42. Devlin JT, Barlow J \& Horton ES (1989) Whole body and regional fuel metabolism during early postexercise recovery. Am J Physiol 256, E167-E172.

43. Gutniak M, Orskov C, Holst JJ, et al. (1992) Antidiabetogenic effect of glucagon-like peptide-1 (7-36)amide in normal subjects and patients with diabetes mellitus. $N$ Engl J Med 326, 1316-1322.

44. Holst JJ \& Deacon CF (2005) Glucagon-like peptide-1 mediates the therapeutic actions of DPP-IV inhibitors. Diabetologia 48, 612-615.

45. Deighton K, Zahra JC \& Stensel DJ (2012) Appetite, energy intake and resting metabolic responses to $60 \mathrm{~min}$ treadmil running performed in a fasted versus a postprandial state. Appetite 58, 946-954.
46. Shorten AL, Wallman KE \& Guelfi KJ (2009) Acute effect of environmental temperature during exercise on subsequent energy intake in active men. Am J Clin Nutr 90, $1215-1221$.

47. Finlayson G, Bryant E, Blundell JE, et al. (2009) Acute compensatory eating following exercise is associated with implicit hedonic wanting for food. Physiol Behav $\mathbf{9 7}$, 62-67.

48. Caudwell P, Gibbons C, Hopkins M, et al. (2011) The influence of physical activity on appetite control: an experimental system to understand the relationship between exercise-induced energy expenditure and energy intake. Proc Nutr Soc 70, 171-180.

49. Blundell JE, Stubbs RJ, Hughes DA, et al. (2003) Cross talk between physical activity and appetite control: does physical activity stimulate appetite? Proc Nutr Soc 62, 651-661.

50. Miller DS, Mumford P \& Stock MJ (1967) Gluttony: 2. Thermogenesis in overeating man. Am J Clin Nutr 20, $1223-1229$

51. Miller DS \& Wise A (1975) Exercise and dietary-induced thermogenesis. Lancet 305, 1290.

52. Welle S (1984) Metabolic responses to a meal during rest and low-intensity exercise. Am J Clin Nutr 40, 990-994.

53. Taboga P, Lazzer S, Fessehatsion R, et al. (2012) Energetics and mechanics of running men: the influence of body mass. Eur J Appl Physiol 112, 4027-4033. 